\title{
Axiomatization of the index of pointedness for closed convex cones
}

\author{
ALFREDO IUSEM $^{1}$ and ALBERTO SEEGER ${ }^{2}$ \\ ${ }^{1}$ Instituto de Matemática Pura e Aplicada \\ Estrada Dona Castorina 110, Jardim Botânico, Rio de Janeiro, Brazil \\ ${ }^{2}$ Univ. of Avignon, Department of Mathematics \\ 33, rue Louis Pasteur, 84000 Avignon, France \\ E-mails: iusp@impa.br / alberto.seeger@univ-avignon.fr
}

\begin{abstract}
Let $\mathcal{C}(H)$ denote the class of closed convex cones in a Hilbert space $H$. One possible way of measuring the degree of pointedness of a cone $K$ is by evaluating the distance from $K$ to the set of all nonpointed cones. This approach has been explored in detail in a previous work of ours. We now go beyond this particular choice and set up an axiomatic background for addressing this issue. We define an index of pointedness over $H$ as being a function $f: \mathcal{C}(H) \rightarrow \mathbb{R}$ satisfying a certain number of axioms. The number $f(K)$ is intended, of course, to measure the degree of pointedness of the cone $K$. Although several important examples are discussed to illustrate the theory in action, the emphasis of this work lies in the general properties that can be derived directly from the axiomatic model.
\end{abstract}

Mathematical subject classification: 47L07, 52A20.

Key words: pointed cone, solid cone, index of pointedness, duality.

\section{Introduction}

Let $H$ be a real Hilbert space with inner product $\langle\cdot, \cdot\rangle$ and associated norm $\|\cdot\|$. For the sake of clarity in the exposition, we always assume that

$$
2 \leq \operatorname{dim} H<\infty .
$$

\#610/04. Received: 19/VII/04. Accepted: 23/IX/04. 
Some of our results can be extended to an infinite dimensional setting, but at the price of a more obscure presentation. The leading role in our discussion is not played by the linear space $H$, but rather by the metric space

$$
\mathcal{C}(H)=\{K \subset H: K \text { is a nonempty closed convex cone }\} .
$$

The metric considered in $\mathrm{C}(H)$ is the usual one, namely

$$
\delta\left(K_{1}, K_{2}\right)=\sup _{\|z\| \leq 1}\left|\operatorname{dist}\left[z, K_{1}\right]-\operatorname{dist}\left[z, K_{2}\right]\right|,
$$

where the notation $\operatorname{dist}[z, K]$ refers to the distance from $z$ to $K$.

The purpose of this work is to elaborate an axiomatic model for dealing with the concept of pointedness. Recall that $K \in \mathcal{C}(H)$ is called pointed if $K \cap-K=\{0\}$. In other words, a cone is pointed if, and only if, it contains no line. Pointedness is a "qualitative" property that has far-reaching consequences. There is no shortage of beautiful theorems in which pointedness plays a prominent role.

Imagine that you have a pointed cone defined in terms of a certain parameter. What happens with the pointedness of the cone if the parameter changes slightly? How much you need to perturb the cone in order to destroy its pointedness? Robustness of a given property is one of the commonest issues addressed by scientists and engineers alike. In the present work we wish to "quantify" the degree of pointedness of a cone. This topic was already addressed in our previous paper [5], but now the orientation is entirely different. Instead of working with a particular measure of pointedness, we set up an axiomatic model from which a more general theory can be developed.

Enough has been said about our motivation. As far as notation is concerned, everything is more or less standard:

$$
\begin{array}{ll}
B_{H}=\{x \in H:\|x\| \leq 1\} & \text { (closed unit ball in } H \text { ) } \\
S_{H}=\{x \in H:\|x\|=1\} & \text { (unit sphere in } H \text { ) } \\
K^{+}=\{y \in H:\langle y, x\rangle \geq 0 \quad \forall x \in K & \text { (dual cone of } K \text { ) } \\
\operatorname{diam}(\Omega)=\sup \{\|u-v\|: u, v \in \Omega\} & (\text { diameter of } \Omega \text { ) } \\
\operatorname{co}(\Omega), \operatorname{cl}(\Omega) & (\text { convex hull of } \Omega, \text { closure of } \Omega \text { ) }
\end{array}
$$




\section{The index of pointedness: an axiomatic formulation}

If $f(K)$ is intended to measure the degree of pointedness of a cone $K \in \mathfrak{C}(H)$, which are the properties that $f$ should satisfy?

- Primo, it is natural to require that $f$ discriminate between the pointed case and the nonpointed one, for instance, $f(K)>0$ if $K$ is pointed, and $f(K)=0$ if $K$ is not pointed.

- Secundo, there is no doubt that a ray $\mathbb{R}_{+} e=\left\{\mu e: \mu \in \mathbb{R}_{+}\right\}(e \neq 0)$ is an extremely pointed object, so it should have the highest possible degree of pointedness. As far as the zero-cone $O_{H}=\{0\}$ is concerned, there are two acceptable strategies: either we take it away from the discussion, or we treat it as a "degenerate" ray (corresponding to $e=0$ ). If the latter strategy is adopted, the degree of pointedness of $O_{H}$ should also be maximal.

- Tertio, changing the orientation of a ray, or, more generally, changing the orientation of a cone, should not affect its degree of pointedness.

This is the bare minimum. To this one could add an extra condition: the degree of pointedness of a cone should diminish if the cone gets bigger. And last, but not the least, if two cones are close to each other, then their corresponding degrees of pointedness should not be too different.

We are now ready to state a formal definition The notation $\operatorname{Isom}(H)$ refers to the space of linear isometries on $H$ (i.e., linear operators $U: H \rightarrow H$ such that $\|U x\|=\|x\| \forall x \in H)$.

Definition 2.1. An index of pointedness on $H$ is a continuous function $f: \mathcal{C}(H) \rightarrow \mathbb{R}$ satisfying the following axioms:

$\left(A_{1}\right)$ minimal pointedness: $f(K)=0$ if and only if $K$ is not pointed;

$\left(A_{2}\right)$ maximal pointedness: $f(K)=1$ if and only if $K$ is either a ray or the zero-cone;

$\left(A_{3}\right)$ invariance property: $f(U(K))=f(K) \forall K \in \mathcal{C}(H), \forall U \in \operatorname{Isom}(H)$;

$\left(A_{4}\right)$ downward monotonicity: $K_{1} \subset K_{2}$ implies $f\left(K_{1}\right) \geq f\left(K_{2}\right)$. 
By convention, the minimal degree of pointedness has been fixed at level 0 , and the maximal one at level 1 . We could work with any other scale and the whole theory would remain essentially the same.

Proposition 2.2. Let $f$ be an index of pointedness on $H$. Then,

$$
\{f(K): K \in \mathcal{C}(H)\}=[0,1] .
$$

Proof. The monotonicity axiom allows us to write

$$
O_{H} \subset K \subset H \quad \Longrightarrow \quad f(H) \leq f(K) \leq f\left(O_{H}\right)
$$

Since $f(H)=0$ and $f\left(O_{H}\right)=1$, one obtains $\{f(K): K \in \mathcal{C}(H)\} \subset[0,1]$. To prove the reverse inclusion, consider an arbitrary unit vector $e \in H$ and define

$$
R(t)=\{x \in H: t\|x\| \leq\langle e, x\rangle\} \quad \forall t \in[0,1] .
$$

A matter of computation yields the estimate

$$
\delta(R(t), R(s))=\left|t \sqrt{1-s^{2}}-s \sqrt{1-t^{2}}\right| \quad \forall t, s \in[0,1] .
$$

Hence, $R:[0,1] \rightarrow \mathcal{C}(H)$ is a continuous path joining the half-space

$$
R(0)=\{x \in H:\langle e, x\rangle \geq 0\}
$$

to the ray $R(1)=\mathbb{R}_{+} e$. As a consequence, the continuous function $t \in[0,1]$ $\mapsto f(R(t))$ takes all the intermediate values between $f(R(0))=0$ and $f(R(1))$ $=1$.

Remark. If the monotonicity requirement $\left(A_{4}\right)$ in Definition 2.1 is replaced by

$$
\left(A_{4}\right)^{\prime} \quad 0 \leq f(K) \leq 1 \quad \forall K \in \mathcal{C}(H),
$$

then one gets a weakened set of axioms. A continuous function $f: \mathcal{C}(H) \rightarrow \mathbb{R}$ satisfying this weakened set of axioms is called a pre-index of pointedness on $H$. The surjectivity result (2) is true also for pre-indices. In fact, the theory of preindices is almost as rich as the theory emerging from the original Definition 2.1. 
The monotonicity requirement $\left(A_{4}\right)$ adds some substance to the discussion, but it is not really the fundamental ingredient.

Definition 2.1 is now going to be scrutinized in detail. As often happens, a good set of axioms leads eventually to a powerful theory which allows people to go far beyond their original expectations. To start with, observe that the class

$$
\chi(H)=\{f: \mathcal{C}(H) \rightarrow \mathbb{R}: f \text { is an index of pointedness on } H\}
$$

is stable with respect to a number of averaging operations:

Proposition 2.3. If $f_{1}, \cdots, f_{m}$ are indices of pointedness on $H$, then any of the following choices corresponds to a new index of pointedness on $H$ :

(a) lower envelope: $\quad f(K)=\min \left\{f_{1}(K), \cdots, f_{m}(K)\right\}$;

(b) upper envelope: $\quad f(K)=\max \left\{f_{1}(K), \cdots, f_{m}(K)\right\}$;

(c) arithmetic average: $\quad f(K)=\frac{f_{1}(K)+\cdots+f_{m}(K)}{m}$;

(d) geometric average: $\quad f(K)=\left[f_{1}(K) \cdots f_{m}(K)\right]^{1 / m}$;

(e) harmonic average: $\quad f(K)=\left\{\frac{\left[f_{1}(K)\right]^{-1}+\cdots+\left[f_{m}(K)\right]^{-1}}{m}\right\}^{-1}$;

(f) log-sigma average: $\quad f(K)=\log \left[\frac{e^{f_{1}(K)}+\cdots+e^{f_{m}(K)}}{m}\right]$.

Proof. Everything can be checked quite easily, so the details are omitted.

One might also think of more sophisticate ways of forming averages, but such a discussion is only of marginal interest. What is perhaps more important to clarify is whether two given members of $\chi(H)$ can be linked together through a simple scaling operation:

$$
f_{2} \sim f_{1} \Longleftrightarrow \exists \gamma \in \Gamma \text { such that } f_{2}=\gamma \circ f_{1},
$$

where the family $\Gamma$ of "scaling functions" is given by

$$
\gamma \in \Gamma \Longleftrightarrow \gamma:[0,1] \rightarrow[0,1] \text { is nondecreasing and surjective. }
$$


Of course, each $\gamma \in \Gamma$ is necessarily continuous and satisfies $\gamma(0)=0$ and $\gamma(1)=1$. One can easily check that (4) is an equivalence relation over $\chi(H)$ (i.e., it is reflexive, symmetric, and transitive). Observe that the indices of pointedness

$$
\sqrt{f(\cdot)}, \quad[f(\cdot)]^{2}, \quad \sin \left[\frac{\pi}{2} f(\cdot)\right], \quad \frac{\log [1+f(\cdot)]}{\log 2}, \cdots
$$

are all equivalent to the index of pointedness $f$ in the sense that they belong to the same equivalence class, namely, the class of $f$.

Most of the interesting indices of pointedness are not just continuous in the ordinary sense, but also Lipschitz continuous. Recall that a function $f: \mathcal{C}(H) \rightarrow$ $\mathbb{R}$ is said to be Lipschitz continuous if the number

$$
\operatorname{lip}(f)=\sup _{K_{1} \neq K_{2}} \frac{\left|f\left(K_{1}\right)-f\left(K_{2}\right)\right|}{\delta\left(K_{1}, K_{2}\right)}
$$

is finite. The function $f: \mathcal{C}(H) \rightarrow \mathbb{R}$ is declared nonexpansive if

$$
\left|f\left(K_{1}\right)-f\left(K_{2}\right)\right| \leq \delta\left(K_{1}, K_{2}\right) \quad \forall K_{1}, K_{2} \in \mathcal{C}(H) .
$$

\section{Three fundamental examples}

Among the different members of $\chi(H)$, some deserve a special mention due to their additional topological properties, or simply because they have an interesting geometric interpretation.

\subsection{The basic approach}

The term "basic" must be understood in a literal sense. Recall that a set $\Omega \subset H$ is called a base for the cone $K \in \mathcal{C}(H)$ if

$$
0 \notin \Omega \quad \text { and } \quad K=\mathbb{R}_{+} \Omega \text {. }
$$

The last condition in (5) is expressed by saying that $\Omega$ generates the cone $K$. As an example of base for $K \neq O_{H}$, one may think of the compact set $K \cap S_{H}$. By taking the convex hull of $K \cap S_{H}$, one gets a convex compact set generating $K$. The trouble with the convexification procedure is that the vector 0 may be 
caught in $\operatorname{co}\left(K \cap S_{H}\right)$. As part of the folklore of the theory of convex cones, one knows that

$$
K \text { is pointed } \Longleftrightarrow 0 \notin \operatorname{co}\left(K \cap S_{H}\right) .
$$

This observation leads us to introduce the number

$$
f_{\star}(K)=\operatorname{dist}\left[0, \operatorname{co}\left(K \cap S_{H}\right)\right]
$$

as a candidate for measuring the degree of pointedness of $K \neq O_{H}$. As far as the zero-cone is concerned, we adopt the convention $f_{\star}\left(O_{H}\right)=1$. The lemma stated below provides a "dual" characterization of (7). The notation

$$
x \in H \mapsto \Psi_{\Omega}^{*}(x)=\sup _{u \in \Omega}\langle u, x\rangle
$$

refers to the support function of $\Omega \subset H$. We assume that the reader is familiar with the main properties of support functions (see, for instance, [4] or [8]). For the sake of convenience, we introduce also the notation

$$
\mathcal{C}_{0}(H)=\mathcal{C}(H) \backslash\left\{O_{H}\right\} .
$$

Lemma 3.1. For any $K \in \mathcal{C}_{0}(H)$, one can write

$$
f_{\star}(K)=\sup _{\|x\| \leq 1}-\Psi_{K \cap S_{H}}^{*}(x) .
$$

Proof. Formula (8) is obtained by applying a standard minimax argument. Observe that

$$
f_{\star}(K)=\inf _{u \in \mathrm{co}}\left(K \cap S_{H}\right) \sup _{x \in B_{H}}\langle u, x\rangle .
$$

Since $\operatorname{co}\left(K \cap S_{H}\right)$ and $B_{H}$ are convex compact sets, von Neumann's minimax theorem allows us to exchange the order of inf and sup. This produces

$$
\begin{aligned}
f_{\star}(K) & =\sup _{x \in B_{H}} \inf _{u \in \operatorname{co}\left(K \cap S_{H}\right)}\langle u, x\rangle \\
& =\sup _{\|x\| \leq 1}-\Psi_{\operatorname{co}\left(K \cap S_{H}\right)}^{*}(-x) \\
& =\sup _{\|x\| \leq 1}-\Psi_{\operatorname{co}\left(K \cap S_{H}\right)}^{*}(x) .
\end{aligned}
$$

The convex hull operation can be dropped from the last term, getting in this way the announced result. 
Before proving that $f_{\star}$ is an index of pointedness, it is helpful to recall some known properties of the Pompeiu-Hausdorff metric

$$
\operatorname{haus}\left[C_{1}, C_{2}\right]=\max \left\{\sup _{a \in C_{1}} \operatorname{dist}\left[a, C_{2}\right], \sup _{b \in C_{2}} \operatorname{dist}\left[b, C_{1}\right]\right\} .
$$

Lemma 3.2. If $C_{1}$ and $C_{2}$ are two nonempty compact sets in $H$, then

$$
\operatorname{haus}\left[C_{1}, C_{2}\right] \geq \operatorname{haus}\left[\operatorname{co}\left(C_{1}\right), \operatorname{co}\left(C_{2}\right)\right]=\sup _{\|x\| \leq 1}\left|\Psi_{C_{1}}^{*}(x)-\Psi_{C_{2}}^{*}(x)\right| .
$$

Proof. The support function characterization of haus $\left[\operatorname{co}\left(C_{1}\right), \operatorname{co}\left(C_{2}\right)\right]$ is well known in the convex analysis community (cf. Theorem 2.18 in Castaing and Valadier [3], or Corollary 3.2.8 in Beer [1]). The inequality in (9) can be found, for instance, in the book by Kisielewicz [7]. Such inequality is almost trivial due to inclusion.

We now are ready to state:

Proposition 3.3. The function $f_{\star}$ is a nonexpansive index of pointedness on $H$.

Proof. Axiom $\left(A_{1}\right)$ is a consequence of (6). Since the function $\|\cdot\|^{2}$ is strictly convex, the equality dist $\left[0, \operatorname{co}\left(K \cap S_{H}\right)\right]=1$ occurs if and only if the set $K \cap S_{H}$ is a singleton. This takes care of $\left(A_{2}\right)$. To check the invariance property $\left(A_{3}\right)$, just notice that

$$
\operatorname{co}\left[U(K) \cap S_{H}\right]=\operatorname{co}\left[U\left(K \cap S_{H}\right)\right]=U\left[\operatorname{co}\left(K \cap S_{H}\right)\right] \quad \forall U \in \operatorname{Isom}(H) .
$$

Monotonicity of $f_{\star}$ is obvious. For proving nonexpansiveness, we rely on Lemmas 3.1 and 3.2. First of all, it must be observed that $\delta$ can be characterized in terms of the Pompeiu-Hausdorff metric, to wit

$$
\delta\left(K_{1}, K_{2}\right)=\operatorname{haus}\left[K_{1} \cap B_{H}, K_{2} \cap B_{H}\right] \quad \forall K_{1}, K_{2} \in \mathcal{C}(H) .
$$

To avoid trivialities, suppose that both cones $K_{1}, K_{2}$ are in $\mathcal{C}_{0}(H)$. In such a case, one can write

$$
\delta\left(K_{1}, K_{2}\right)=\operatorname{haus}\left[K_{1} \cap S_{H}, K_{2} \cap S_{H}\right],
$$


and, with the help of Lemma 3.2, one gets

$$
-\Psi_{K_{1} \cap S_{H}}^{*}(x) \leq-\Psi_{K_{2} \cap S_{H}}^{*}(x)+\delta\left(K_{1}, K_{2}\right) \quad \forall x \in B_{H} .
$$

By taking the supremum over $B_{H}$ and applying Lemma 3.1, one obtains

$$
f_{\star}\left(K_{1}\right) \leq f_{\star}\left(K_{2}\right)+\delta\left(K_{1}, K_{2}\right) .
$$

It suffices now to exchange the roles of $K_{1}$ and $K_{2}$ to complete the proof.

\subsection{The hemi-diametral approach}

It is based on the evaluation of the number

$$
r(K)=\frac{1}{2} \operatorname{diam}\left(K \cap S_{H}\right),
$$

which corresponds to half the diameter of $K \cap S_{H}$. Observe that the mapping $K \mapsto r(K)$ ranges from 0 (when $K$ is a ray) to 1 (when $K$ is nonpointed). Since $r$ has not the right monotonicity, we suggest considering instead

$$
f_{[1]}(K)=1-r(K) \text {. }
$$

In fact, one can also consider the more general expression

$$
f_{[p]}(K)=\left[1-[r(K)]^{p}\right]^{1 / p},
$$

with $p \in[1, \infty$ [ being chosen arbitrarily. The case $p=2$ is of special relevance as we shall see in due course. The term (10) makes sense only if $K \neq O_{H}$, so, by convention, one sets $f_{[p]}\left(O_{H}\right)=1$. As shown in the next lemma, the function $r$ has a fairly good continuity behavior.

Lemma 3.4. For any $K_{1}, K_{2} \in \mathcal{C}_{0}(H)$, one has the Lipschitz estimate

$$
\left|\operatorname{diam}\left(K_{1} \cap S_{H}\right)-\operatorname{diam}\left(K_{2} \cap S_{H}\right)\right| \leq 2 \delta\left(K_{1}, K_{2}\right) .
$$


Proof. This result is probably known. In order to prove (11), we start by obtaining an alternative characterization of the diameter function. For a nonempty bounded set $\Omega \subset H$, one can write

$$
\operatorname{diam}(\Omega)=\sup _{u, v \in \Omega} \sup _{x \in B_{H}}\langle x, u-v\rangle=\sup _{x \in B_{H}} \sup _{u, v \in \Omega}\{\langle x, u\rangle+\langle x,-v\rangle\},
$$

producing in this way

$$
\operatorname{diam}(\Omega)=\sup _{x \in B_{H}}\left\{\Psi_{\Omega}^{*}(x)+\Psi_{-\Omega}^{*}(x)\right\} .
$$

We will apply this general formula to the particular choices $\Omega=K_{1} \cap S_{H}$ and $\Omega=K_{2} \cap S_{H}$. By Lemma 3.2, we know already that

$$
\Psi_{K_{1} \cap S_{H}}^{*}(x) \leq \Psi_{K_{2} \cap S_{H}}^{*}(x)+\delta\left(K_{1}, K_{2}\right) \quad \forall x \in B_{H},
$$

as well as,

$$
\Psi_{-\left(K_{1} \cap S_{H}\right)}^{*}(x) \leq \Psi_{-\left(K_{2} \cap S_{H}\right)}^{*}(x)+\delta\left(-K_{1},-K_{2}\right) \quad \forall x \in B_{H} .
$$

Summing up and observing that $\delta\left(-K_{1},-K_{2}\right)=\delta\left(K_{1}, K_{2}\right)$, one gets

$$
\begin{aligned}
\Psi_{K_{1} \cap S_{H}}^{*}(x)+\Psi_{-\left(K_{1} \cap S_{H}\right)}^{*}(x) \leq & \Psi_{K_{2} \cap S_{H}}^{*}(x)+\Psi_{-\left(K_{2} \cap S_{H}\right)}^{*}(x) \\
& +2 \delta\left(K_{1}, K_{2}\right) \quad \forall x \in B_{H} .
\end{aligned}
$$

To complete the proof, we just need to take the supremum with respect to $x \in B_{H}$.

Without further ado, we state:

Proposition 3.5. For each $p \in\left[1, \infty\left[\right.\right.$, the function $f_{[p]}$ is an index of pointedness on $\mathrm{H}$.

Proof. The diameter of $K \cap S_{H}$ equals 2 if and only if $K$ contains two opposite unit vectors. The set $K \cap S_{H}$ is a singleton if and only if $K$ is a ray. These statements take care of Axioms $\left(A_{1}\right)$ and $\left(A_{2}\right)$, respectively. The invariance property $\left(A_{3}\right)$ follows from

$\operatorname{diam}\left[U(K) \cap S_{H}\right]=\operatorname{diam}\left[U\left(K \cap S_{H}\right)\right]=\operatorname{diam}\left[K \cap S_{H}\right] \quad \forall U \in \operatorname{Isom}(H)$. Monotonicity of $f_{[p]}$ is obvious. Lemma 3.4 yields the continuity of $K \rightarrow$ $\operatorname{diam}\left(K \cap S_{H}\right)$ as function defined over metric subspace $\mathcal{C}_{0}(H)$. This fact guarantees, in turn, the continuity of $f_{[p]}$ over the whole metric space $\mathcal{C}(H)$. 
Proposition 3.6. For any $p, q \in\left[1, \infty\left[\right.\right.$, the indices $f_{[p]}$ and $f_{[q]}$ are equivalent.

Proof. For passing from $f_{[p]}$ to $f_{[q]}$, consider the scaling function

$$
t \in[0,1] \mapsto \gamma(t)=\left[1-\left(1-t^{p}\right)^{q / p}\right]^{1 / q} .
$$

It is a mere routine to check that $\gamma \in \Gamma$.

As mentioned before, the choice $p=2$ is of special relevance. A simple computation shows that

$$
f_{[2]}(K)=\sqrt{1-[r(K)]^{2}}
$$

admits the equivalent characterization

$$
f_{[2]}(K)=\sqrt{\frac{1+\cos \theta_{\max }(K)}{2}}=\cos \left(\frac{\theta_{\max }(K)}{2}\right),
$$

where $\theta_{\max }(K)$ denotes the largest angle that can be formed by picking up two unit vectors in $K$, that is to say

$$
\theta_{\max }(K)=\sup _{u, v \in K \cap S_{H}} \arccos \langle u, v\rangle .
$$

Due to the formula (13), we refer to $f_{[2]}(K)$ as the angular index of pointedness of $K$ (we reserve the term "angular" for the index $f_{[2]}$, but is is clear from Proposition 3.6 that any hemi-diametral index $f_{[p]}$ can be expressed in terms of the function $\theta_{\max }$ ).

The equivalence between (12) and (13) can be proven in a rather easy way by exploiting the general identity

$$
\|u-v\|^{2}=2(1-\langle u, v\rangle) \quad \forall u, v \in S_{H} .
$$

Below we provide two additional characterizations of the function $f_{[2]}$. Recall that the gap between two nonempty sets $A, B \subset H$ is defined as the number

$$
\operatorname{gap}[A, B]=\inf _{a \in A, b \in B}\|a-b\| .
$$

General ingredients on the theory of gaps can be found, for instance, in the book of Beer [1]. 
Lemma 3.7. For any $K \in \mathfrak{C}_{0}(H)$, one has

$$
f_{[2]}(K)=\frac{1}{2} \operatorname{gap}\left[K \cap S_{H},-K \cap S_{H}\right],
$$

and also

$$
f_{[2]}(K)=\inf _{\|z\|=1} \max \{\operatorname{dist}[z, K], \operatorname{dist}[-z, K]\},
$$

Proof. Formula (14) is easier to prove. By definition of a gap, one has

$$
\operatorname{gap}\left[K \cap S_{H},-K \cap S_{H}\right]=\inf _{u \in K \cap S_{H}, w \in-K \cap S_{H}}\|u-w\|=\inf _{u, v \in K \cap S_{H}}\|u+v\| .
$$

By working out the last expression, one arrives at

$$
\operatorname{gap}\left[K \cap S_{H},-K \cap S_{H}\right]=\inf _{u, v \in K \cap S_{H}} 2 \sqrt{\frac{1+\langle u, v\rangle}{2}}=2 f_{[2]}(K) .
$$

Formula (15) is proven in our work [6]. The proof, which is quite long and technical, doesn't deserve to be reproduced here. Observe, incidentally, that (15) applies also to the zero cone, the convention $f_{[2]}\left(O_{H}\right)=1$ being in force. $\square$

Remark. As done in [6], it is interesting to observe that

$$
\left\{\begin{array}{c}
\forall K \in \mathcal{C}_{0}(H), \text { there is a unit vector } z \in H \\
\text { such that } f_{[2]}(K)=\operatorname{dist}[z, K]=\operatorname{dist}[-z, K] .
\end{array}\right.
$$

When $\mathrm{K}$ is not a ray, such a vector $z$ can be constructed, for instance, by normalizing $u-v$, with $u, v \in K \cap S_{H}$ satisfying $\|u-v\|=\operatorname{diam}\left(K \cap S_{H}\right)$.

We now return to the analysis of the family $\left\{f_{[p]}: p \in[1, \infty[\}\right.$ of hemidiametral indices. As shown in the next theorem, nonexpansiveness can be obtained only for special choices of $p$. Before stating such a result, a preliminary lemma is in order. Observe that $f_{[p]}$ admits the representation

$$
f_{[p]}(K)=\varphi_{p}(r(K)) \quad \forall K \in \mathcal{C}_{0}(H),
$$

with $\varphi_{p}:[0,1] \rightarrow[0,1]$ being defined by $\varphi_{p}(\tau)=\left[1-\tau^{p}\right]^{1 / p}$. Formula (16) applies also to $K=O_{H}$ if one adopts the convention $r\left(O_{H}\right)=0$. According to Lemma 3.4, the function $r$ is nonexpansive. As far as $\varphi_{p}$ is concerned, one has: 
Lemma 3.8. Let $p \in] 1,2\left[\right.$. Then, there exist $\left.\tau_{p} \in\right] 0,1[$ and a positive constant $L_{p}$ such that

(a) $\left|\varphi_{p}(t)-\varphi_{p}(s)\right| \leq L_{p}|t-s| \quad \forall t, s \in\left[0, \tau_{p}\right]$;

(b) $\left|\varphi_{p}(t)-\varphi_{p}(s)\right| \leq\left|\varphi_{2}(t)-\varphi_{2}(s)\right| \quad \forall t, s \in\left[\tau_{p}, 1\right]$;

(c) $\left|\varphi_{p}(t)-\varphi_{p}(s)\right| \leq\left|\varphi_{2}(t)-\varphi_{2}(s)\right|+L_{p}|t-s| \quad \forall t, s \in[0,1]$.

Proof. For proving the part (a), observe that the derivative

$$
\varphi_{p}^{\prime}(\tau)=-\left[\frac{\tau}{\left(1-\tau^{p}\right)^{1 / p}}\right]^{p-1}
$$

is well defined over $\left[0, \tau_{p}\right]$, and

$$
L_{p}=\sup _{\tau \in\left[0, \tau_{p}\right]}\left|\varphi_{p}^{\prime}(\tau)\right|<\infty .
$$

The above remark applies to any choice of $\left.\tau_{p} \in\right] 0,1[$. For proving the part (b), we take $\tau_{p}$ so that

$$
\varphi_{p}^{\prime}(\tau) \geq \varphi_{2}^{\prime}(\tau) \quad \forall \tau \in\left[\tau_{p}, 1[.\right.
$$

To check that such a $\tau_{p}$ exists, we write (17) in the form

$$
\left[\frac{\tau}{\left(1-\tau^{p}\right)^{1 / p}}\right]^{p-1} \leq \frac{\tau}{\sqrt{1-\tau^{2}}},
$$

or, what is equivalent,

$$
\tau^{4-2 p} \geq \frac{1-\tau^{2}}{\left(1-\tau^{p}\right)^{2(p-1) / p}} .
$$

Obviously, the left-hand side of (18) goes to 1 as $\tau \rightarrow 1^{-}$, while an application of l'Hôspital's rule establishes that the right-hand side goes to 0 as $\tau \rightarrow 1^{-}$. Thus the inequality in (18) is valid for $\tau$ close enough to 1 . Once (17) has been established for a suitable $\tau_{p}$, one completes the proof of (b) by using an integration argument. The details are omitted because the integration mechanism is illustrated in the proof of (c). For proving the part (c), we consider only the 
difficult case in which $t$ and $s$ are not on the same side with respect to $\tau_{p}$. Take for instance $0 \leq s<\tau_{p}<t<1$. Observe that

$$
\begin{aligned}
\left|\varphi_{p}(t)-\varphi_{p}(s)\right| & =-\left[\varphi_{p}(t)-\varphi_{p}(s)\right] \\
& =-\int_{s}^{t} \varphi_{p}^{\prime}(\tau) d \tau \\
& =\int_{s}^{\tau_{p}}-\varphi_{p}^{\prime}(\tau) d \tau+\int_{\tau_{p}}^{t}-\varphi_{p}^{\prime}(\tau) d \tau .
\end{aligned}
$$

But

$$
\int_{s}^{\tau_{p}}-\varphi_{p}^{\prime}(\tau) d \tau \leq L_{p}\left(\tau_{p}-s\right) \leq L_{p}|t-s|
$$

and

$$
\int_{\tau_{p}}^{t}-\varphi_{p}^{\prime}(\tau) d \tau \leq \int_{\tau_{p}}^{t}-\varphi_{2}^{\prime}(\tau) d \tau \leq-\left[\varphi_{2}(t)-\varphi_{2}\left(\tau_{p}\right)\right] \leq\left|\varphi_{2}(t)-\varphi_{2}(s)\right| .
$$

The proof of the lemma is thus complete.

Theorem 3.9. The following statements are true:

(a) the indices $f_{[1]}$ and $f_{[2]}$ are nonexpansive;

(b) for any $p \in] 1,2\left[\right.$, the index $f_{[p]}$ is Lipschitz continuous;

(c) for any $p>2$, the index $f_{[p]}$ is not Lipschitz continuous.

\section{Proof.}

- Part (a). Nonexpansiveness of $f_{[1]}$ is a direct consequence of Lemma 3.4. Nonexpansiveness of $f_{[2]}$ follows from the characterization (15) and the general inequality

$\operatorname{dist}\left[z, K_{1}\right] \leq \operatorname{dist}\left[z, K_{2}\right]+\delta\left(K_{1}, K_{2}\right) \quad \forall z \in S_{H}, \forall K_{1}, K_{2} \in \mathcal{C}(H)$.

- Part (b). To handle the case $p \in] 1,2[$, we exploit Lemma 3.8 and the representation formula (16). Consider two arbitrary cones $K_{1}, K_{2} \in \mathcal{C}_{0}(H)$. If $r\left(K_{1}\right)$ and $r\left(K_{2}\right)$ fall both in the interval [0, $\left.\tau_{p}\right]$, then Lemma 3.7(a) yields

$$
\left|f_{[p]}\left(K_{2}\right)-f_{[p]}\left(K_{1}\right)\right| \leq L_{p}\left|r\left(K_{2}\right)-r\left(K_{1}\right)\right| \leq L_{p} \delta\left(K_{2}, K_{1}\right) .
$$


If $r\left(K_{1}\right)$ and $r\left(K_{2}\right)$ fall both in the interval $\left[\tau_{p}, 1\right]$, then we use Lemma 3.8(b) to obtain

$$
\left|f_{[p]}\left(K_{2}\right)-f_{[p]}\left(K_{1}\right)\right| \leq\left|f_{[2]}\left(K_{2}\right)-f_{[2]}\left(K_{1}\right)\right| \leq \delta\left(K_{2}, K_{1}\right) .
$$

If $r\left(K_{1}\right)$ and $r\left(K_{2}\right)$ are not in the same side with respect to $\tau_{p}$, then Lemma 3.8(c) does the job. One gets in this case

$$
\begin{aligned}
\left|f_{[p]}\left(K_{2}\right)-f_{[p]}\left(K_{1}\right)\right| & \leq\left|f_{[2]}\left(K_{2}\right)-f_{[2]}\left(K_{1}\right)\right|+L_{p}\left|r\left(K_{2}\right)-r\left(K_{1}\right)\right| \\
& \leq\left(1+L_{p}\right) \delta\left(K_{2}, K_{1}\right) .
\end{aligned}
$$

- Part (c). To handle the case $p>2$, consider the cone $R(t)$ given by (3). As a matter of computation, one gets

$$
f_{[p]}(R(t))=\left[1-\left(\sqrt{1-t^{2}}\right)^{p}\right]^{1 / p} \quad \forall t \in[0,1],
$$

and, therefore,

$$
\begin{aligned}
\operatorname{lip}\left(f_{[p]}\right) & \geq \frac{\left|f_{[p]}(R(t))-f_{[p]}(R(0))\right|}{\delta(R(t), R(0))} \\
& =\frac{1}{t}\left[1-\left(\sqrt{1-t^{2}}\right)^{p}\right]^{1 / p}
\end{aligned}
$$

for any $t \in] 0,1[$. Observe that the term on the right-hand side of (19) goes to $\infty$ as $t \rightarrow 0^{+}$.

\subsection{The metric approach}

We cannot avoid mentioning the function $f_{\delta}: \mathcal{C}(H) \rightarrow[0,1]$ defined by

$$
f_{\delta}(K)=\inf _{Q \in \mathcal{M}(H)} \delta(Q, K) .
$$

The number $f_{\delta}(K)$ represents the distance from $K$ to the set

$$
\mathcal{M}(H)=\{Q \in \mathcal{C}(H): Q \text { is not pointed }\} .
$$

Since $\mathcal{M}(H)$ is a compact set in the metric space $(\mathcal{C}(H), \delta)$, the infimum in (20) is actually attained. In our work [5], we refer to the number $f_{\delta}(K)$ as the radius of pointedness of $K$. The reason for this name is that

$$
f_{\delta}(K)=\sup \left\{r \in[0,1]: U_{r}(K) \subset \mathcal{P}(H)\right\}
$$


corresponds to the radius of the largest ball

$$
U_{r}(K)=\{Q \in \mathcal{C}(H): \delta(Q, K)<r\}
$$

centered at $K$ and contained in the set $\mathcal{P}(H)=\mathcal{C}(H) \backslash \mathcal{M}(H)$ of pointed cones.

Proposition 3.10. The function $f_{\delta}$ is a nonexpansive pre-index of pointedness on $H$.

Proof. See the reference [5].

Proposition 3.11. Among all the nonexpansive pre-indices of pointedness on $H, f_{\delta}$ is the largest one (in the pointwise sense).

Proof. Take an arbitrary $K \in \mathcal{C}(H)$. For any nonexpansive function $f: \mathcal{C}(H)$ $\rightarrow \mathbb{R}$, one can write

$$
f(K) \leq f(Q)+\delta(Q, K) \quad \forall Q \in \mathcal{C}(H),
$$

and, in particular,

$$
f(K) \leq \inf _{Q \in \mathcal{M}(H)}\{f(Q)+\delta(Q, K)\} .
$$

If $f$ vanishes over $\mathcal{M}(H)$, the above inequality reduces to $f(K) \leq f_{\delta}(K)$.

It is not clear whether $f_{\delta}$ satisfies the monotonicity requirement $\left(A_{4}\right)$. Partial evidence leads us to conjecture that $f_{\delta}$ is indeed monotone, but we are not yet in a position of giving a definite answer to this delicate issue.

\section{Basic index versus angular index}

Both indices share many properties in common, but they do behave differently with respect to dimensional issues. To start with, we state:

Proposition 4.1. For any $K \in \mathcal{C}(H)$, one has $f_{\star}(K) \leq f_{[2]}(K)$. 
Proof. Take $K \neq\{0\}$ and write

$$
\begin{aligned}
f_{\star}(K) & =\inf _{x \in \operatorname{co}\left(K \cap S_{H}\right)}\|x\| \\
& \leq \inf _{u, v \in K \cap S_{H}}\left\|\frac{u+v}{2}\right\| \\
& =\inf _{u, v \in K \cap S_{H}} \sqrt{\frac{1+\langle u, v\rangle}{2}}=f_{[2]}(K) .
\end{aligned}
$$

This proves the announced inequality.

The above proof hides, in fact, a general result:

Lemma 4.2. If $K \in \mathcal{C}(H)$ contains $m$ mutually obtuse unit vectors, then $f_{\star}(K) \leq 1 / \sqrt{m}$.

Proof. According to the hypothesis, one can find $m$ unit vectors $a_{1}, \cdots, a_{m}$ in $K$ such that $\left\langle a_{i}, a_{j}\right\rangle \leq 0 \forall i \neq j$. Hence,

$$
\begin{aligned}
{\left[f_{\star}(K)\right]^{2} } & =\inf _{x \in \operatorname{co}\left(K \cap S_{H}\right)}\|x\|^{2} \leq\left\|\frac{a_{1}+\cdots+a_{m}}{m}\right\|^{2} \\
& =\frac{1}{m^{2}}\left\{\sum_{i=1}^{m}\left\|a_{i}\right\|^{2}+2 \sum_{i<j}\left\langle a_{i}, a_{j}\right\rangle\right\} .
\end{aligned}
$$

Since the $a_{i}$ 's have unit length and are mutually obtuse, one gets $\left[f_{\star}(K)\right]^{2}$ $\leq 1 / m$.

With the help of Lemma 4.2 one can easily show that $f_{\star} \neq f_{[2]}$, that is to say, $f_{\star}(K)<f_{[2]}(K)$ for some $K \in \mathcal{C}(H)$.

Example 4.3. Take $H=\mathbb{R}^{n}$ with $n \geq 3$. Clearly, $\theta_{\max }\left(\mathbb{R}_{+}^{n}\right)=\pi / 2$ and $f_{[2]}\left(\mathbb{R}_{+}^{n}\right)=1 / \sqrt{2}$. On the other hand, the positive orthant $\mathbb{R}_{+}^{n}$ is a closed convex cone containing $n$ mutually orthogonal unit vectors. So,

$$
f_{\star}\left(\mathbb{R}_{+}^{n}\right) \leq 1 / \sqrt{n} \leq 1 / \sqrt{3}<f_{[2]}\left(\mathbb{R}_{+}^{n}\right) .
$$

The exact value of $f_{\star}\left(\mathbb{R}_{+}^{n}\right)$ will be given in Proposition 4.9. As suggested by Example 4.3, the index $f_{\star}$ is ill-conditioned if one works in a space of large 
dimension: the degree of pointedness of the corresponding positive orthant is almost zero. This "strange" behavior of $f_{\star}$ becomes even worse in an infinitedimensional setting: $f_{\star}$ is no longer an index of pointedness!

Example 4.4. In the Hilbert space $H=\ell^{2}$ of square summable real sequences, consider the pointed cone

$$
\ell_{+}^{2}=\left\{x \in \ell^{2}: x_{k} \geq 0 \quad \forall k \in \mathbb{N}\right\}
$$

and the canonical vectors $a_{1}=(1,0,0, \cdots), a_{2}=(0,1,0, \cdots), \ldots$ Since the first $n$ canonical vectors lie in $\ell_{+}^{2}$ and are mutually orthogonal, it follows that

$$
f_{\star}\left(\ell_{+}^{2}\right) \leq 1 / \sqrt{n}
$$

But this argument applies to an arbitrary $n$, so $f_{\star}\left(\ell_{+}^{2}\right)=0$. In other words, $f_{\star}$ does not satisfy the axiom $\left(A_{1}\right)$. This fact should not be too surprising after all: one knows that the characterization (6) of pointedness holds only if the underlying space is finite dimensional.

The next proposition has to do with the particular case of a finitely generated cone, that is to say, a cone expressible as

$$
K=\left\{\mu_{1} g_{1}+\cdots+\mu_{m} g_{m}: \mu \in \mathbb{R}_{+}^{m}\right\} .
$$

Without loss of generality one may assume that the generators $g_{1}, \cdots, g_{m} \in \mathbb{R}^{n}$ are vectors of unit length. An upper bound for $f_{\star}(K)$ is obtained easily by minimizing a convex quadratic form over the elementary simplex

$$
\Sigma_{m}=\left\{\mu \in \mathbb{R}_{+}^{m}: \mu_{1}+\cdots+\mu_{m}=1\right\} .
$$

Proposition 4.5. Let $K \subset \mathbb{R}^{n}$ be the finitely generated cone given by (21). Denote by $G$ the $n \times m$ matrix whose columns are the generators $g_{1}, \cdots, g_{m} \in$ $S_{\mathbb{R}^{n}}$. Then,

$$
\left[f_{\star}(K)\right]^{2} \leq \inf _{\mu \in \Sigma_{m}}\left\langle\mu, G^{T} G \mu\right\rangle
$$


Proof. It is enough to observe that $f_{\star}(K) \leq\|G \mu\|$ for every $\mu \in \Sigma_{m}$.

One of the reasons for introducing the index $f_{\star}$ is that its computational cost is not too high. As indicated in the proof of Lemma 3.1, one has

$$
f_{\star}(K)=\sup _{\|x\| \leq 1} \rho_{K}(x),
$$

with

$$
\rho_{K}(x)=\inf _{u \in K \cap S_{H}}\langle u, x\rangle .
$$

Solving the inner minimization problem (23) amounts to finding a unit vector in $K$ which forms the largest angle with respect to the given $x$. The best choice for $x$ is obtained by solving the outer maximization problem (22).

Definition 4.6. A centroid of $K \in \mathcal{C}_{0}(H)$ is a maximizer of $\rho_{K}$ over $B_{H}$, that is to say, a vector in

$$
\operatorname{ctr}(K)=\left\{x \in B_{H}: \rho_{K}(x)=f_{\star}(K)\right\} .
$$

Since $\rho_{K}: H \rightarrow \mathbb{R}$ is a concave function, the set $\operatorname{ctr}(K)$ is nonempty compact and convex. This set turns out to be a singleton if the cone $K$ is pointed:

Proposition 4.7. A pointed cone $K \in \mathcal{C}_{0}(H)$ admits exactly one centroid. Moreover, the centroid lies in $K \cap S_{H}$.

Proof. Consider first the case of an arbitrary $K \in \mathcal{C}_{0}(H)$, be it pointed or not. We claim that

$$
x_{0} \in \operatorname{ctr}(K) \Longleftrightarrow\left\{\begin{array}{l}
\text { there is a vector } y \in N\left[x_{0}, B_{H}\right] \text { such that } \\
y \in \operatorname{co}\left(K \cap S_{H}\right) \text { and }\left\langle y, x_{0}\right\rangle=\rho_{K}\left(x_{0}\right),
\end{array}\right.
$$

where

$$
N\left[x_{0}, B_{H}\right]= \begin{cases}\{0\} & \text { if }\left\|x_{0}\right\|<1, \\ \mathbb{R}_{+} x_{0} & \text { if }\left\|x_{0}\right\|=1\end{cases}
$$

corresponds to the normal cone to $B_{H}$ at $x_{0}$. Observe that $x_{0} \in \operatorname{ctr}(K)$ if and only if $x_{0}$ minimizes $-\rho_{K}$ over $B_{H}$. Since we are dealing with a convex minimization problem, the standard first-order optimality condition is both necessary 
and sufficient (cf. Theorem 27.4 in [8]). So,

$$
\operatorname{ctr}(K)=\left\{x_{0} \in H: 0 \in \partial\left(-\rho_{K}\right)\left(x_{0}\right)+N\left[x_{0}, B_{H}\right]\right\}
$$

with $\partial$ denoting the subdifferential operator in the sense of convex analysis. For obtaining (24), it is enough to observe that

$$
\begin{aligned}
-\partial\left(-\rho_{K}\right)\left(x_{0}\right) & =\partial \Psi_{K \cap S_{H}}^{*}\left(-x_{0}\right) \\
& =\left\{y \in H: y \in \operatorname{co}\left(K \cap S_{H}\right) \text { and }\left\langle y, x_{0}\right\rangle=\rho_{K}\left(x_{0}\right)\right\},
\end{aligned}
$$

the last equality being a consequence of a general calculus rule for computing the subdifferential of a support function (cf. Corollary 23.5.3 in [8]). Consider now the particular case in which $K$ is pointed. According to (24), the inequality $\left\|x_{0}\right\|<1$ must be ruled out because $0 \notin \operatorname{co}\left(K \cap S_{H}\right)$. Hence, the centroids of $K$ lie necessarily in $S_{H}$. By writing

$$
\left.\left.y=\beta x_{0} \quad \text { with } \quad \beta \in\right] 0,1\right],
$$

one sees that $x_{0}=\beta^{-1} y \in K$. Summarizing, we have proven that $\operatorname{ctr}(K)$ is a nonempty convex set contained in $K \cap S_{H}$. This implies, of course, that $\operatorname{ctr}(K)$ contains exactly one element.

As shown by the proof of Proposition 4.7, the centroid of a nonzero pointed cone $K$ can be characterized as follows:

$$
x_{0} \text { is the centroid of } K \Longleftrightarrow\left\{\begin{array}{cc}
\left\|x_{0}\right\|=1, & \left.\left.\rho_{K}\left(x_{0}\right) \in\right] 0,1\right], \\
& \text { and } \\
\rho_{K}\left(x_{0}\right) x_{0} \in \operatorname{co}\left(K \cap S_{H}\right) .
\end{array}\right.
$$

For a revolution cone, for instance, the centroid corresponds to the so-called axis of revolution. In fact, one has:

Proposition 4.8. Consider a revolution cone $K=\{x \in H:\|x\| \cos \vartheta \leq$ $\langle e, x\rangle\}$ with axis of revolution $e \in S_{H}$ and angle of revolution $\vartheta \in[0, \pi / 2[$. Then,

$$
f_{\star}(K)=\rho_{K}(e)=\cos \vartheta .
$$


Proof. Pick up an arbitrary $b \in S_{H}$ such that $\langle b, e\rangle=0$. Since

$$
u=(\cos \vartheta) e+(\sin \vartheta) b \text { and } v=(\cos \vartheta) e-(\sin \vartheta) b
$$

belong to $K \cap S_{H}$, one has

$$
f_{\star}(K) \leq\|(u+v) / 2\|=\|(\cos \vartheta) e\|=\cos \vartheta .
$$

On the other hand,

$$
f_{\star}(K) \geq \rho_{K}(e)=\inf _{x \in K \cap S_{H}}\langle e, x\rangle=\cos \vartheta .
$$

Another instance where the centroid can be easily computed is that of a positive orthant:

Proposition 4.9. The centroid of the positive orthant $\mathbb{R}_{+}^{n}$ is $x_{0}=$ $n^{-1 / 2}(1,1, \cdots, 1)$, and

$$
f_{\star}\left(\mathbb{R}_{+}^{n}\right)=\rho_{\mathbb{R}_{+}^{n}}\left(x_{0}\right)=1 / \sqrt{n} .
$$

Proof. Clearly $\left\|x_{0}\right\|=1$. It is geometrically clear that the infimal-value

$$
\rho_{\mathbb{R}_{+}^{n}}\left(x_{0}\right)=\inf _{u \in \mathbb{R}_{+}^{n},\|u\|=1} \frac{u_{1}+\cdots+u_{n}}{\sqrt{n}}
$$

is attained at any of the generators of $\mathbb{R}_{+}^{n}$. This allows us to check the right-hand side of (25), and obtain the formula (26).

We end this section by showing that the basic index is essentially different from the angular index.

Proposition 4.10. When $\operatorname{dim} H \geq 3$, the indices $f_{\star}$ and $f_{[2]}$ are not equivalent.

Proof. Let $H=\mathbb{R}^{n}$, with $n \geq 3$. The positive orthant $\mathbb{R}_{+}^{n}$ and the ice-cream cone

$$
\Lambda_{n}=\left\{x \in \mathbb{R}^{n}:\left[x_{1}^{2}+\cdots+x_{n-1}^{2}\right]^{1 / 2} \leq x_{n}\right\}
$$

have both a maximal angle equal to $\pi / 2$. Thus,

$$
f_{[2]}\left(\Lambda_{n}\right)=f_{[2]}\left(\mathbb{R}_{+}^{n}\right)=1 / \sqrt{2} .
$$


On the other hand,

$$
f_{\star}\left(\Lambda_{n}\right)=1 / \sqrt{2} \text { but } f_{\star}\left(\mathbb{R}_{+}^{n}\right)=1 / \sqrt{n}<1 / \sqrt{2} \text {. }
$$

This rules out the possibility of finding a scaling function $\gamma \in \Gamma$ such that $f_{\star}=\gamma \circ f_{[2]}$.

\section{Normalization}

Starting with an arbitrary index of pointedness, one can construct a new one by using a simple scaling procedure. If we are lucky enough, we could find a suitable scaling function bringing our initial index to a sort of "normal" form. This raises the question of what must be understood by a normal index. There are different ways of answering this question, everything depending on what we have in mind when we speak about normalizing an index.

Recall that the index of a nonpointed cone has been fixed at the minimum level 0 , whereas the index of a ray has been fixed at the maximum level 1. So, what about an intermediate situation? What kind a cone could be considered as a good compromise between a nonpointed cone and a ray? Which one should be the corresponding index of such a cone?

To answer to these questions, we arrange the cones according to their maximal angle. On the one hand side, the case $\theta_{\max }(K)=0$ occurs when $K$ is a ray, and, on the other hand, the condition $\theta_{\max }(K)=\pi$ indicates that $K$ is not pointed. An interesting intermediate situation is $\theta_{\max }(K)=\pi / 2$. One can easily check that

$$
\begin{aligned}
& \theta_{\max }(K)=\frac{\pi}{2} \quad \Longleftrightarrow \quad K \text { is acute and contains a pair of } \\
& \text { orthogonal unit vectors. }
\end{aligned}
$$

That $K \in \mathcal{C}(H)$ is acute simply means that $\langle u, v\rangle \geq 0, \forall u, v \in K$. A cone $K \in \mathcal{C}(H)$ as in (27) is said to be perpendicular. We are now ready to introduce the concept of normality.

Definition 5.1. One says that $f \in \chi(H)$ is normal if

$$
f(K)=\sqrt{2} / 2 \text { for every perpendicular } K \in \mathcal{C}(H) .
$$


If there is a scaling function $\gamma \in \Gamma$ such that $\gamma \circ f$ is normal, then $\mathrm{f}$ is declared normalizable.

In other words, an index of pointedness is normalizable if and only if it is constant over the class of perpendicular cones. By way of example, we mention that the basic index $f_{\star}$ is not normalizable: as shown in the proof of Proposition $4.10, f_{\star}$ takes different values over the class of self-dual cones (which is contained in the class of perpendicular cones). The angular index $f_{[2]}$ behaves much better in this respect:

Proposition 5.2. For any $K \in \mathcal{C}(H)$, one has:

(a) $f_{[2]}(K) \geq \sqrt{2} / 2$ if and only if $K$ is acute;

(b) $f_{[2]}(K) \leq \sqrt{2} / 2$ if and only if $K$ contains a pair of orthogonal unit vectors.

Hence, the index $f_{[2]}$ is normal.

Proof. It follows directly from the characterization (13).

Corollary 5.3. The pre-index $f_{\delta}$ is normal.

Proof. As shown in [6], the functions $f_{\delta}$ and $f_{[2]}$ coincide over the class of perpendicular cones. It suffices then to apply Proposition 5.2. We observe, incidentally, that $f_{\delta}$ and $f_{[2]}$ coincide over a larger class of cones, namely,those having a maximal angle less than or equal to 120 degrees.

Proposition 5.2 can be extended in the following way:

Proposition 5.4. Suppose that the index $f \in \chi(H)$ is of the angular-type, meaning that

$$
\theta_{\max }\left(K_{1}\right)=\theta_{\max }\left(K_{2}\right) \text { implies } f\left(K_{1}\right)=f\left(K_{2}\right) .
$$

Then, $f$ is normalizable. 
Proof. Consider any $\theta \in[0, \pi]$. If $f$ is of the angular-type, then $f$ is constant over the level set

$$
\left\{\theta_{\max }=\theta\right\}=\left\{K \in \mathcal{C}(H): \theta_{\max }(K)=\theta\right\} .
$$

In particular, $f$ is constant over $\left\{\theta_{\max }=\pi / 2\right\}$, the class of perpendicular cones.

Remark. Any index $f_{[p]}$ from the hemi-diametral family is of the angular-type, so it is normalizable.

\section{Dualization}

Recall that a cone $K \in \mathcal{C}(H)$ is said to be solid if its topological interior is nonempty. In a finite dimensional setting, solidity is a dual concept with respect to pointedness:

$$
K \text { is pointed } \Leftrightarrow K^{+} \text {is solid. }
$$

A simple proof of this equivalence can be found, for instance, in the book by Berman [2]. Inspired by (29), we dualize the concept of pointedness index in the following manner:

Definition 6.1. An index of solidity on $H$ is a continuous function $g: \mathcal{C}(H) \rightarrow$ $\mathbb{R}$ satisfying the following axioms:

( $\left.A_{1}\right)$ minimal solidity: $g(K)=0$ if and only if $K$ is not solid;

$\left(A_{2}\right)$ maximal solidity: $g(K)=1$ if and only if $K$ contains a halfspace;

$\left(A_{3}\right)$ invariance property: $g(U(K))=g(K) \forall K \in \mathcal{C}(H), \forall U \in \operatorname{Isom}(H)$;

( $\left.A_{4}\right)$ upward monotonicity: $K_{1} \subset K_{2}$ implies $g\left(K_{1}\right) \leq g\left(K_{2}\right)$.

There is no need to explore Definition 6.1 in detail because measuring the degree of solidity of a cone $K$ is essentially the same job as measuring the degree 
of pointedness of its polar $K^{+}$. This idea is stated properly in the following proposition, where we use the notation

$$
\begin{aligned}
& \Phi: \mathcal{C}(H) \mapsto \mathcal{C}(H) \\
& K \mapsto \Phi(K)=K^{+}
\end{aligned}
$$

to indicate the polarity mapping. A celebrated theorem by Walkup and Wets [9] asserts that $\Phi$ is an isometry over the metric space $(\mathcal{C}(H), \delta)$, i.e.

$$
\delta\left(K_{1}^{+}, K_{2}^{+}\right)=\delta\left(K_{1}, K_{2}\right) \quad \forall K_{1}, K_{2} \in \mathcal{C}(H) .
$$

Proposition 6.2. The polarity mapping $\Phi: \mathcal{C}(H) \mapsto \mathcal{C}(H)$ relates the concepts introduced in Definitions 2.1 and 6.1 as follows:

(a) if $f$ is an index of pointedness, then $f \circ \Phi$ is an index of solidity;

(b) if $g$ is an index of solidity, then $g \circ \Phi$ is an index of pointedness.

Proof. Everything is straightforward. It is a matter of exploiting the well known properties of the mapping $\Phi$.

As pointed out to us by Adrian Lewis (personal communication), a possible way of measuring the degree of solidity of a cone $K$ is in terms of the expression

$$
g_{\star}(K)=\sup \left\{r:\|x\|=1, r \geq 0, x+r B_{H} \subset K\right\} .
$$

This corresponds to the radius of the largest ball contained in $K$ and centered at a unit vector. One assumes, of course, that $K \neq H$, otherwise the convention $g_{\star}(H)=1$ is in order. It turns out that (30) defines an index of solidity in the sense of Definition 6.1:

Proposition 6.3. The function $g_{\star}$ given by (30) is a nonexpansive index of solidity. In fact,

$$
g_{\star}(K)=f_{\star}\left(K^{+}\right) \quad \forall K \in \mathcal{C}(H),
$$

with $f_{\star}$ denoting the basic index of pointedness. 
Proof. Suppose that $K \in \mathfrak{C}(H)$ is not the whole space. Write (30) in the form

$$
g_{\star}(K)=\sup _{\|x\|=1} \sup _{\substack{r \geq 0 \\ x+r B_{H} \subset K}} r
$$

and observe that

$$
x+r B_{H} \subset K \Longleftrightarrow r\|y\| \leq\langle x, y\rangle \forall y \in K^{+} \Longleftrightarrow r \leq \inf _{\substack{y \|=1 \\ y \in K^{+}}}\langle x, y\rangle .
$$

This proves that

$$
g_{\star}(K)=\sup _{\|x\|=1} \inf _{\substack{\|y\|=1 \\ y \in K^{+}}}\langle x, y\rangle .
$$

The above expression remains unchanged if $x$ ranges over the unit ball $B_{H}$ (and not just over the unit sphere $S_{H}$ ). Also, no change occurs if the infimum is taken over convex hull of $K^{+} \cap S_{H}$ (and not just over $K^{+} \cap S_{H}$ ). As we did in Lemma 3.1, we apply von Neumann's minimax theorem to conclude that

$$
g_{\star}(K)=\sup _{x \in B_{H}} \inf _{y \in \operatorname{co}\left(K^{+} \cap S_{H}\right)}\langle x, y\rangle=\inf _{y \in \operatorname{co}\left(K^{+} \cap S_{H}\right)} \sup _{x \in B_{H}}\langle x, y\rangle=f_{\star}\left(K^{+}\right) .
$$

The proof of (31) is thus complete. Propositions 3.3 and 6.2 do the rest of the job.

Observe that the formula (31) can be written in the equivalent form

$$
f_{\star}(K)=g_{\star}\left(K^{+}\right) \quad \forall K \in \mathcal{C}(H),
$$

that is to say, the basic index of pointedness of a cone $K$ can be computed by evaluating the index of solidity $g_{\star}$ at $K^{+}$. For illustrating this general principle, we examine next the particular case of a nondegenerate elliptic cone in $\mathbb{R}^{n} \times \mathbb{R}$. Such term refers to a set of the form

$$
\mathcal{E}(A)=\left\{(u, s) \in \mathbb{R}^{n} \times \mathbb{R}: \sqrt{u^{t} A u} \leq s\right\},
$$

with $A$ being a positive definite symmetric matrix of order $n \times n$. The symbol $u^{t}$ denotes, of course, the transpose of the column vector $u$. 
Proposition 6.4. Let $A$ be a positive definite symmetric matrix of order $n \times n$. Then,

$$
f_{\star}(\mathcal{E}(A))=\sqrt{\frac{\lambda_{\min }(A)}{1+\lambda_{\min }(A)}} \quad \text { and } \quad g_{\star}(\mathcal{E}(A))=\sqrt{\frac{1}{1+\lambda_{\max }(A)}},
$$

with $\lambda_{\min }(A)$ and $\lambda_{\max }(A)$ denoting, respectively, the smallest and largest eigenvalue of $A$.

Proof. Due to the formula (32) and the general identity $[\mathcal{E}(A)]^{+}=\mathcal{E}\left(A^{-1}\right)$, we need to evaluate only the term $g_{\star}(\mathcal{E}(A))$. To do this, we look at the largest ball centered at $\bar{x}=(0, \cdots, 0,1) \in \mathbb{R}^{n} \times \mathbb{R}$ and contained in $\mathcal{E}(A)$. For this it suffices to find the closest point to $\bar{x}$ in the boundary of $\mathcal{E}(A)$ : the distance from such point to $\bar{x}$ will be the radius of such largest ball. Since the boundary of $\mathcal{E}(A)$ is given by

$$
\operatorname{bd}[\mathcal{E}(A)]=\left\{(u, s) \in \mathbb{R}^{n} \times \mathbb{R}: \sqrt{u^{t} A u}=s\right\},
$$

we must solve the minimization problem

$$
\min u^{t} u+(s-1)^{2} \quad \text { with }(u, s) \in \mathbb{R}^{n} \times \mathbb{R} \text { s.t. } u^{t} A u-s^{2}=0 .
$$

If $(u, s)$ is a solution to (33), then there is a Lagrange multiplier $\lambda \in \mathbb{R}$ such that

$$
u-\lambda A u=0, \quad s-1+\lambda s=0 .
$$

Notice that $\lambda$ is an eigenvalue of $A^{-1}, u$ is a corresponding eigenvector, and $s=(1+\lambda)^{-1}$. Clearly

$$
\begin{gathered}
(1+\lambda)^{-2}=s^{2}=u^{t} A u=\lambda^{-1} u^{t} u, \\
(s-1)^{2}=\left(\frac{1}{1+\lambda}-1\right)^{2}=\left(\frac{\lambda}{1+\lambda}\right)^{2},
\end{gathered}
$$

from where one obtains

$$
u^{t} u+(s-1)^{2}=\frac{\lambda}{(1+\lambda)^{2}}+\frac{\lambda^{2}}{(1+\lambda)^{2}}=\frac{1}{1+\lambda^{-1}} .
$$


We conclude that the optimal value $r^{2}$ of (33) is of the form $\left(1+\lambda^{-1}\right)^{-1}$, with $\lambda>0$ being an eigenvalue of $A^{-1}$. One can easily see that the smallest value of

$$
r=\left(1+\lambda^{-1}\right)^{-1 / 2}
$$

is obtained by choosing $\lambda=\lambda_{\min }\left(A^{-1}\right)$. One gets in this way the estimate

$$
g_{\star}(\mathcal{E}(A)) \geq\left[1+\left[\lambda_{\min }\left(A^{-1}\right)\right]^{-1}\right]^{-1 / 2}=\left[1+\lambda_{\max }(A)\right]^{-1 / 2} .
$$

But, on the other hand, one can also write

$$
g_{\star}(\mathcal{E}(A)) \leq g_{[2]}(\mathcal{E}(A))=\left[1+\lambda_{\max }(A)\right]^{-1 / 2} .
$$

The inequality in (34) follows from Propositions 4.1 and 6.3, while the equality in (34) is a result established in [5].

In the next proposition we provide an expression for the index of solidity $g_{[p]}$ which is obtained by dualizing the index of pointedness $f_{[p]}$.

Proposition 6.5. $\quad$ Let $_{[p]}: \mathcal{C}(H) \rightarrow \mathbb{R}$ be the function defined by the expression

$$
g_{[p]}(K)=\left[1-[m(K)]^{p}\right]^{1 / p},
$$

with

$$
m(K)=\sup _{\|z\|=1} \min \{\operatorname{dist}[z, K], \operatorname{dist}[-z, K]\} .
$$

Then, $g_{[p]}$ is an index of solidity. In fact,

$$
g_{[p]}(K)=f_{[p]}\left(K^{+}\right) \quad \forall K \in \mathcal{C}(H) .
$$

Proof. We need to prove the equality (36). The case $K=H$ is trivial and therefore it is left aside. Consider then an arbitrary $K \neq H$. Proving (36) is, of course, the same as checking the equality

$$
m(K)=r\left(K^{+}\right)
$$

To do this, we exploit Lemma 3.7 and the well-known Pithagorean rule

$$
\operatorname{dist}^{2}[z, K]+\operatorname{dist}^{2}\left[z, K^{-}\right]=\|z\|^{2} \quad \forall z \in H,
$$


with $K^{-}=-K^{+}$standing for the negative polar cone of $K$. Indeed, one has

$$
\begin{aligned}
r^{2}\left(K^{+}\right) & =1-f_{[2]}^{2}\left(K^{+}\right) \\
& =1-f_{[2]}^{2}\left(K^{-}\right) \\
& =1-\left[\inf _{\|z\|=1} \max \left\{\operatorname{dist}\left[z, K^{-}\right], \operatorname{dist}\left[-z, K^{-}\right]\right\}\right]^{2} \\
& =1-\inf _{\|z\|=1} \max \left\{1-\operatorname{dist}^{2}[z, K], 1-\operatorname{dist}^{2}[-z, K]\right\} .
\end{aligned}
$$

A simple algebraic manipulation shows that the last term corresponds to $m^{2}(K)$. The proof is then complete.

As far as the dualization of the pre-index of pointedness $f_{\delta}$ is concerned, we have shown in [5] the formula

$$
g_{\delta}(K)=f_{\delta}\left(K^{+}\right) \quad \forall K \in \mathcal{C}(H),
$$

with $g_{\delta}$ being the distance function to the set of non-solid cones, that is to say,

$$
g_{\delta}(K)=\inf \{\delta(Q, K): Q \in \mathcal{C}(H) \text { non-solid }\} .
$$

\section{Interlude: a tale of maximal angles}

Recall that $\theta_{\max }(K)$ denotes the maximal angle that can be formed by picking up two unit vectors in $K$. The symbol $\theta_{\max }\left(K^{+}\right)$is defined, of course, in a similar way. The question we would like to answer in this section has a very strong geometric flavour:

$$
\left\{\begin{array}{l}
\text { is there any relationship between the } \\
\text { maximal angles } \theta_{\max }(K) \text { and } \theta_{\max }\left(K^{+}\right) ?
\end{array}\right.
$$

It would be very surprising if nobody has thought about this question before. Anyway, we have been unable to find a trace of this issue in the existing literature. Anwering (38) would enable us to establish a link between the angular index of a cone and the angular index of its dual. For convenience, we reformulate (38) in a seemingly different manner:

$$
\left\{\begin{array}{l}
\text { is there any relationship between the diameter } \\
\text { of } K \cap S_{H} \text { and the diameter of } K^{+} \cap S_{H}
\end{array}\right.
$$

No extra comments are needed. Here is what we get: 
Lemma 7.1. Assume that $K \in \mathcal{C}(H)$ is neither the zero-cone, nor the whole space $H$. Then,

$$
\left[\operatorname{diam}\left(K \cap S_{H}\right)\right]^{2}+\left[\operatorname{diam}\left(K^{+} \cap S_{H}\right)\right]^{2} \geq 4 .
$$

Proof. If both $K \cap S_{H}$ and $K^{+} \cap S_{H}$ have a diameter greater than or equal to $\sqrt{2}$, then the result holds trivially. We assume from now that this is not the case. Suppose, for instance, that $\operatorname{diam}\left(K \cap S_{H}\right)<\sqrt{2}$. Due to (12) and (13), this assumption entails

$$
\langle u, v\rangle>0 \quad \forall u, v \in K .
$$

If $K$ is a ray, then $\operatorname{diam}\left(K \cap S_{H}\right)=0, \operatorname{diam}\left(K^{+} \cap S_{H}\right)=2$, and (39) holds. Assume that $K$ is not a ray, and take $u, v \in K \cap S_{H}$ such that $\operatorname{diam}\left(K \cap S_{H}\right)=$ $\|u-v\|>0$. Let $M=\operatorname{span}\{u, v\}$ be the two-dimensional linear subspace spanned by $u$ and $v$. Consider the vectors

$$
y=\frac{u-\langle u, v\rangle v}{\sqrt{1-\langle u, v\rangle^{2}}}, \quad z=\frac{v-\langle u, v\rangle u}{\sqrt{1-\langle u, v\rangle^{2}}} .
$$

By construction, $y \in M$ and $z \in M$ enjoy the following properties:

$$
\begin{gathered}
\langle y, u\rangle \geq 0, \quad\langle z, v\rangle \geq 0, \\
\langle z, u\rangle=\langle y, v\rangle=0, \\
\langle y, z\rangle=-\langle u, v\rangle, \\
\|y\|=\|z\|=1 .
\end{gathered}
$$

Everything can be checked in a rather easy way. We will prove that $y, z$ belong to $K^{+}$. To do this, it suffices to show that $\langle y, x\rangle \geq 0,\langle z, x\rangle \geq 0$ for all $x \in K \cap S_{H}$. So, take $x \in K \cap S_{H}$. We find the projection $P_{M}(x)$ of $x$ onto $M$ by solving a simple minimization problem in two variables. We get

$$
P_{M}(x)=\lambda u+\mu v,
$$

with coefficients $\lambda, \mu \in \mathbb{R}$ given by

$$
\lambda=\frac{\langle u, x\rangle-\langle x, v\rangle\langle u, v\rangle}{1-\langle u, v\rangle^{2}}, \quad \mu=\frac{\langle v, x\rangle-\langle x, u\rangle\langle u, v\rangle}{1-\langle u, v\rangle^{2}} .
$$


We claim that $\lambda, \mu \geq 0$. By (40) we know that $\langle x, u\rangle,\langle x, v\rangle \in] 0,1]$ and $\langle u, v\rangle \in$ ]0, 1 . Since $\|u-v\|=\operatorname{diam}\left(K \cap S_{H}\right)$, we have that $\langle u, v\rangle \leq\langle u, x\rangle$ and $\langle u, v\rangle \leq\langle v, x\rangle$. Thus,

$$
\begin{aligned}
& 0<\langle x, v\rangle\langle u, v\rangle \leq\langle u, v\rangle \leq\langle u, x\rangle, \\
& 0<\langle x, u\rangle\langle u, v\rangle \leq\langle u, v\rangle \leq\langle v, x\rangle .
\end{aligned}
$$

This establishes our claim. We now use the orthogonality property

$$
\left\langle w, x-P_{M}(x)\right\rangle=0 \quad \forall w \in M
$$

of the projection $P_{M}(x)$, to obtain finally

$$
\begin{gathered}
\langle y, x\rangle=\left\langle y, P_{M}(x)\right\rangle=\lambda\langle y, u\rangle+\mu\langle y, v\rangle \geq 0, \\
\langle z, x\rangle=\left\langle z, P_{M}(x)\right\rangle=\lambda\langle z, u\rangle+\mu\langle z, v\rangle \geq 0 .
\end{gathered}
$$

Since $x$ was an arbitrary vector of $K \cap S_{H}$, we have indeed established that $y, z$ belong to $K^{+}$. It follows that $\operatorname{diam}\left(K^{+} \cap S\right) \geq\|y-z\|$, and therefore

$$
\begin{aligned}
{\left[\operatorname{diam}\left(K \cap S_{H}\right)\right]^{2}+\left[\operatorname{diam}\left(K^{+} \cap S_{H}\right)\right]^{2} } & \geq\|u-v\|^{2}+\|y-z\|^{2} \\
& =4-2(\langle u, v\rangle+\langle y, z\rangle)=4,
\end{aligned}
$$

completing the proof in this way.

Everything is now ready for answering the question stated at the beginning of the section.

Theorem 7.2 [First law of maximal angles]. Assume that $K \in \mathcal{C}(H)$ is neither the zero-cone, nor the whole space H. Then,

$$
\pi \leq \theta_{\max }(K)+\theta_{\max }\left(K^{+}\right) .
$$

Proof. Proving this inequality is a matter of exploiting Lemma 7.1 and the general identity

$$
\left[\operatorname{diam}\left(K \cap S_{H}\right)\right]^{2}=2\left[1-\cos \theta_{\max }(K)\right] .
$$


Theorem 7.3 [Second law of maximal angles]. Assume that $\operatorname{dim} H \geq 3$. Then, for any pair $\left(\theta_{1}, \theta_{2}\right) \in[0, \pi] \times[0, \pi]$ such that $\pi \leq \theta_{1}+\theta_{2}$, there is a cone $K \in \mathcal{C}(H)$ satisfying

$$
\theta_{\max }(K)=\theta_{1} \quad \text { and } \quad \theta_{\max }\left(K^{+}\right)=\theta_{2} .
$$

Proof. Since $K \mapsto \theta_{\max }(K)$ is continuous over the compact metric space $(\mathcal{C}(H), \delta)$, it suffices to consider the case $\left.\left(\theta_{1}, \theta_{2}\right) \in\right] 0, \pi[\times] 0, \pi[$. For convenience, we work in the space $H=\mathbb{R}^{n} \times \mathbb{R}$. The integer $n$ is taken, of course, greater than or equal to 2 . As candidate for achieving (41), we consider a nondegenerate elliptic cone $\mathcal{E}(A)$ in $\mathbb{R}^{n} \times \mathbb{R}$. As shown in our previous work [5], one has

$$
\begin{aligned}
\theta_{\max }(\mathcal{E}(A)) & =\arccos \left[\frac{\lambda_{\min }(A)-1}{\lambda_{\min }(A)+1}\right], \\
\theta_{\max }\left([\mathcal{E}(A)]^{+}\right) & =\arccos \left[\frac{1-\lambda_{\max }(A)}{1+\lambda_{\max }(A)}\right] .
\end{aligned}
$$

For proving the theorem, it is enough to construct a matrix $A$ such that

$$
\lambda_{\min }(A)=\frac{1+\cos \theta_{1}}{1-\cos \theta_{1}}, \quad \lambda_{\max }(A)=\frac{1-\cos \theta_{2}}{1+\cos \theta_{2}} .
$$

Such a construction is possible provided the inequality

$$
\frac{1+\cos \theta_{1}}{1-\cos \theta_{1}} \leq \frac{1-\cos \theta_{2}}{1+\cos \theta_{2}}
$$

holds. Observe that (42) is equivalent to

$$
\cos \theta_{1}+\cos \theta_{2} \leq 0,
$$

the later inequality holding trivially because the pair $\left.\left(\theta_{1}, \theta_{2}\right) \in\right] 0, \pi[\times] 0, \pi[$ satisfies $\pi \leq \theta_{1}+\theta_{2}$.

\section{Sub-unitarian indices}

Theorems 7.2 and 7.3 could be merged into a single one that provides an estimate for the range of the function

$$
K \mapsto\left(\theta_{\max }(K), \theta_{\max }\left(K^{+}\right)\right) .
$$


In the same way as $\theta_{\max }(K)$ and $\theta_{\max }\left(K^{+}\right)$are related to each other, we expect there a link between the degree of pointedness of $K$ and the degree of pointedness of $K^{+}$. The question addressed in this section is that of estimating the region

$$
\Omega(f)=\left\{\left(f(K), f\left(K^{+}\right)\right): K \in \mathcal{C}(H)\right\}
$$

of all "configurations" that can be produced with a given index $f \in \chi(H)$. It must be observed that $\Omega(f)$ does not fill the whole square $[0,1] \times[0,1]$ because the configuration

$$
\left(f(K), f\left(K^{+}\right)\right)=(1,1)
$$

can never occur. In principle, it is possible to have both $f(K)$ and $f\left(K^{+}\right)$very close to 1 for a given $K \in \mathrm{C}(H)$, but this would mean that $f$ is somehow badly conditioned. A scaling procedure may be necessary to correct such an anomaly. A favourable class of indices is singled out in the next definition.

Definition 8.1. One says that $f \in \chi(H)$ is sub-unitarian if

$$
[f(K)]^{2}+\left[f\left(K^{+}\right)\right]^{2} \leq 1 \quad \forall K \in \mathcal{C}(H) .
$$

The term fully sub-unitarian is reserved for the case

$$
\Omega(f)=\left\{(r, s) \in \mathbb{R}_{+} \times \mathbb{R}_{+}: r^{2}+s^{2} \leq 1\right\} .
$$

Examples of sub-unitarian indices are not difficult to construct. An important example is displayed next.

Proposition 8.2. The angular index $f_{[2]}$ is sub-unitarian. If the underlying space $H$ has dimension at least 3 , then $f_{[2]}$ is fully sub-unitarian.

Proof. Assume that $K \in \mathrm{C}(H)$ is neither the zero-cone, nor the whole space $H$. As a consequence of the first law of maximal angles, one gets

$$
\cos \theta_{\max }(K)+\cos \theta_{\max }\left(K^{+}\right) \leq 0 .
$$

By using the relations

$$
\left[f_{[2]}(K)\right]^{2}=\frac{1+\cos \theta_{\max }(K)}{2}, \quad\left[f_{[2]}\left(K^{+}\right)\right]^{2}=\frac{1+\cos \theta_{\max }\left(K^{+}\right)}{2},
$$


one obtains

$$
\left[f_{[2]}(K)\right]^{2}+\left[f_{[2]}\left(K^{+}\right)\right]^{2}=1+\frac{1}{2}\left[\cos \theta_{\max }(K)+\cos \theta_{\max }\left(K^{+}\right)\right] \leq 1 .
$$

In this way, we have proven that

$$
\Omega\left(f_{[2]}\right) \subset\left\{(r, s) \in \mathbb{R}_{+} \times \mathbb{R}_{+}: r^{2}+s^{2} \leq 1\right\}
$$

For getting the reverse inclusion, it is enough to work out the example of an elliptic cone as done in the proof of Theorem 7.3.

Corollary 8.3. For any $p \in\left[1,2\left[\right.\right.$, the hemi-diametral index $f_{[p]}$ is subunitarian. By contrast, $f_{[p]}$ is not sub-unitarian if $p>2$.

Proof. For any $p \in[1,2[$, one has

$$
f_{[p]}(K) \leq f_{[2]}(K) \quad \forall K \in \mathcal{C}(H) .
$$

The sub-unitarian character of $f_{[2]}$ implies that of $f_{[p]}$. Consider now the case $p>2$. Pick up any self-dual cone $K$ in $H$. Since

$$
\operatorname{diam}\left(K \cap S_{H}\right)=\operatorname{diam}\left(K^{+} \cap S_{H}\right)=\sqrt{2},
$$

the number

$$
\left[f_{[p]}(K)\right]^{2}+\left[f_{[p]}\left(K^{+}\right)\right]^{2}=2\left[1-(1 / 2)^{p / 2}\right]^{2 / p}
$$

is strictly greater than 1 .

Corollary 8.4. The basic index $f_{\star}$ is sub-unitarian. If the underlying space $H$ has dimension at least 3 , then $f_{\star}$ is fully sub-unitarian.

Proof. For the first part of the corollary, combine Propositions 4.1 and 8.2. For the second part, use Proposition 8.2 and the fact that $f_{\star}$ coincides with $f_{[2]}$ over the class of nondegenerate elliptic cones. An explicit expression for $f_{\star}(\mathcal{E}(A))$ is given in Proposition 6.4. As can be seen from the proof of Theorem 8.3 in [5], the same expression applies also to $f_{[2]}(\mathcal{E}(A))$. 


\section{Rotational invariance}

The purpose of this section is to show that the angular index of pointedness $f_{\star}$ can be characterized in terms of a certain property that we call rotational invariance. A slightly different version of this property can be used to characterize the angular index $f_{[2]}$. Some of the results stated in this section were suggested to us by an anonymous referee to whom we are very grateful.

Before introducing the concept of rotational invariance, recall that a revolution cone in $H$ is a set of the form

$$
\operatorname{rev}(\vartheta, e)=\{x \in H:\|x\| \cos \vartheta \leq\langle e, x\rangle\},
$$

with $e \in S_{H}$ refered to as the axis of revolution, and $\vartheta \in[0, \pi / 2]$ refered to as the angle of revolution. The degree of pointedness of a revolution cone depends uniquely on the angle of revolution. More precisely,

Lemma 9.1. If $f$ is an index of pointedness on $H$, then there is a scaling function $\gamma \in \Gamma$ such that

$$
f(\operatorname{rev}(\vartheta, e))=\gamma(\cos \vartheta) \quad \forall e \in S_{H}, \vartheta \in[0, \pi / 2] .
$$

Such function $\gamma$ is unique and given by

$$
\gamma(t)=f(\operatorname{rev}(\arccos t, e)) \quad \forall t \in[0,1],
$$

with $e \in S_{H}$ being chosen arbitrarily.

Proof. The second part of the lemma follows from the first one. For proving the representation formula (43), we rely on the axioms defining an index of pointedness. The invariance axiom $\left(A_{3}\right)$ implies that $f(\operatorname{rev}(\vartheta, e))$ depends uniquely on the parameter $\vartheta$, that is to say, there is a function $F:[0, \pi / 2] \rightarrow \mathbb{R}$ such that

$$
f(\operatorname{rev}(\vartheta, e))=F(\vartheta) .
$$

The function $F$ is necessarily continuous because it corresponds to the composition of the continuous functions $f$ and $\vartheta \rightarrow \operatorname{rev}(\vartheta, e)$. The above equality can be transformed into (43) by taking

$$
\gamma=F \circ \arccos .
$$


The minimal pointedness axiom $\left(A_{1}\right)$ implies that $\gamma(0)=0$. The maximal pointedness axiom $\left(A_{2}\right)$ implies that $\gamma(1)=1$. Finally, the monotonicity axiom $\left(A_{4}\right)$ implies that $\gamma$ is nondecreasing. In short, $\gamma$ is a scaling function as required.

Next we introduce two different revolution cones that can be associated to a given pointed cone.

Definition 9.2. Let $K \in \mathcal{C}_{0}(H)$ be a pointed cone. The rotational envelope of $K$, which we denote by $\operatorname{rot} K$, is the pointed revolution cone obtained by rotating $K$ around its centroid. The companion of $K$, which we denote by $\operatorname{com} K$, is the pointed revolution cone that has the same centroid as $K$ and the same maximal angle as $K$.

It is not difficult to see that $\operatorname{com} K$ admits the characterization

$$
\begin{aligned}
\operatorname{com} K & =\operatorname{rev}\left(\frac{\theta_{\max }(K)}{2}, e_{K}\right) \\
& =\left\{x \in H:\|x\| \cos \left(\frac{\theta_{\max }(K)}{2}\right) \leq\left\langle e_{K}, x\right\rangle\right\},
\end{aligned}
$$

with $e_{K}$ denoting the centroid of $K$. As far as the characterization of $\operatorname{rot} K$ is concerned, observe that

$$
\arccos \rho\left(e_{K}\right)=\sup _{x \in K \cap S_{H}} \arccos \left\langle e_{K}, x\right\rangle
$$

corresponds to the largest angle with respecto to $e_{K}$ that can be formed by picking up a unit vector in $K$. Rotating $K$ around $e_{K}$ produces then the revolution cone

$$
\operatorname{rot} K=\operatorname{rev}\left(\arccos \rho\left(e_{K}\right), e_{K}\right)=\left\{x \in H:\|x\| \rho\left(e_{K}\right) \leq\left\langle e_{K}, x\right\rangle\right\} .
$$

As a general rule, $\operatorname{com} K$ and $\operatorname{rot} K$ are different objects. Although the following result is very easy to prove, it deserves to be properly recorded.

Proposition 9.3. For a pointed cone $K \in \mathcal{C}_{0}(H)$, the following two conditions are equivalent:

(a) com $K$ and rot $K$ coincide;

(b) $f_{[2]}(K)=f_{\star}(K)$. 
Proof. In view of (45) and (46), condition (a) amount to saying that

$$
\cos \left(\frac{\theta_{\max }(K)}{2}\right)=\rho\left(e_{K}\right) .
$$

This equality is, of course, the same as the one given in (b).

Recall that from the very definition of the companion of a cone, one has

$$
\theta_{\max }(\operatorname{com} K)=\theta_{\max }(K) .
$$

Preservation of the maximal angle is a nice property, but it doesn't imply preservation of the degree of pointedness, unless, of course, one uses an index of pointedness which is equivalent to $f_{[2]}$. This idea is made more precise in the next theorem.

Theorem 9.4. For an index of pointedness $f \in \chi(H)$, the following two conditions are equivalent:

(a) $f$ is rotationally invariant in the sense that $f(\operatorname{com} K)=f(K)$ for any pointed cone $K \in \mathrm{e}_{0}(H)$;

(b) $f$ is equivalent to the angular index $f_{[2]}$.

Proof. That (b) implies (a) follows directly from (47) and the representation formula (13) of $f_{[2]}$. To prove the reverse implication, suppose that $f \in \chi(H)$ is a rotationally invariant index of pointedness. Let $\gamma \in \Gamma$ be the scaling function whose existence and characterization is given by Lemma 9.1. For any pointed cone $K \in \mathfrak{C}_{0}(H)$, one has

$$
\begin{aligned}
f(K) & =f(\operatorname{com} K)=f\left(\operatorname{rev}\left(\frac{\theta_{\max }(K)}{2}, e_{K}\right)\right) \\
& =\gamma\left(\cos \left(\frac{\theta_{\max }(K)}{2}\right)\right)=\gamma\left(f_{[2]}(K)\right) .
\end{aligned}
$$

This proves that $f$ is equivalent to the angular index $f_{[2]}$.

Theorem 9.4 admits an analogous formulation having the basic index of pointedness $f_{\star}$ as main protagonist. 
Theorem 9.5. For an index of pointedness $f \in \chi(H)$, the following two conditions are equivalent:

(a) $f$ is rotationally invariant in the sense that $f(\operatorname{rot} K)=f(K)$ for any pointed cone $K \in \mathrm{C}_{0}(H)$;

(b) $f$ is equivalent to the basic index $f_{\star}$.

Proof. One clearly has

$$
f_{\star}(\operatorname{rot} K)=f_{\star}\left(\operatorname{rev}\left(\arccos \rho\left(e_{K}\right), e_{K}\right)=\rho\left(e_{K}\right)=f_{\star}(K),\right.
$$

which shows that (b) implies (a). For proving the reverse implication, one exploits Lemma 9.1 as in the proof of Theorem 9.4. This time one gets

$$
f(K)=f(\operatorname{rot} K)=f\left(\operatorname{rev}\left(\arccos \rho\left(e_{K}\right), e_{K}\right)=\gamma\left(\rho\left(e_{K}\right)\right)=\gamma\left(f_{\star}(K)\right) .\right.
$$

\section{Conclusions}

In this work we have introduced the concept of index of pointedness by following an axiomatic approach. Several examples were given to illustrate the general theory.

Among the different particular examples, the angular index of pointedness $f_{[2]}$ deserves a special mention because it enjoys a number of convenient properties. Indeed, $f_{[2]}$ is nonexpansive, normal and sub-unitarian.

\begin{tabular}{|c|c|c|c|c|}
\hline & nonexpansive & normal & normalizable & sub-unitarian \\
\hline$f_{[1]}$ & yes & no & yes & yes \\
\hline$\left.f_{[p]}, p \in\right] 1,2[$ & $?$ & no & yes & yes \\
\hline$f_{[2]}$ & yes & yes & yes & yes \\
\hline$\left.f_{[p]}, p \in\right] 2, \infty[$ & no & no & yes & no \\
\hline$f_{\star}$ & yes & no & no & yes \\
\hline$f_{\delta}$ & yes & yes & yes & $?$ \\
\hline
\end{tabular}

Table 1 - Indices and pre-indices of pointedness.

The main drawback of the basic index $f_{\star}$ is not being normalizable. Said in a crude manner, there is no way of scaling this index so as to obtain a measure 
of pointedness that is well conditioned with respect to the dimension of the underlying space.

Our main purpose was lying down a general theory for quantifying the degree of pointedness of a convex cone. The subjet under consideration is quite broad and admits several ramifications. Some questions were left open because it is impossible to solve in a single work all the difficulties encountered in the road. For instance, a very challenging question is checking whether or not the function $f_{\delta}$ is monotone. Recall that the monotonicity requirement appears in the very definition of an index of pointedness. A less important question is evaluating the Lipschitz constant $\operatorname{lip}\left(f_{[p]}\right)$ of the hemi-diametral index $f_{[p]}$ when $\left.p \in\right] 1,2[$.

Acknowledgements. The authors are very grateful to an anonymous referee whose constructive remarks have improved the presentation of the paper. Section 9 is inspired in his report.

\section{REFERENCES}

[1] G. Beer, Topologies on Closed and Closed Convex Sets, Kluwer Academic Publ., Dordrecht, 1993.

[2] A. Berman, Cones, Matrices and Mathematical Programming, Lect. Notes in Economics and Mathematical Systems, 79 (1973), Springer-Verlag, Berlin.

[3] C. Castaing and M. Valadier, Convex Analysis and Measurable Multifunctions, Lect. Notes in Mathematics, 580 (1977), Springer-Verlag, Berlin.

[4] J.B. Hiriart-Urruty and C. Lemarechal, Convex Analysis ans Minimization Algorithms, Vol I, Springer-Verlag, Berlin, 1993.

[5] A. Iusem and A. Seeger, Measuring the degree of pointedness of a closed convex cone: a metric approach, to appear in Mathematische Nachrichten.

[6] A. Iusem and A. Seeger, Computing the radius of pointedness of a closed convex cone, to appear in Mathematical Programming.

[7] M. Kisielewicz, Differential Inclusions and Optimal Control, Kluwer Academic Publ., Dordrecht, 1991.

[8] R.T. Rockafellar, Convex Analysis, Princeton Univ. Press, Princeton, New Jersey, 1970.

[9] D.W. Walkup and R.J.B. Wets, Continuity of some convex-cone-valued mappings, Proc. Amer. Math. Soc., 18 (1967), 229-235. 